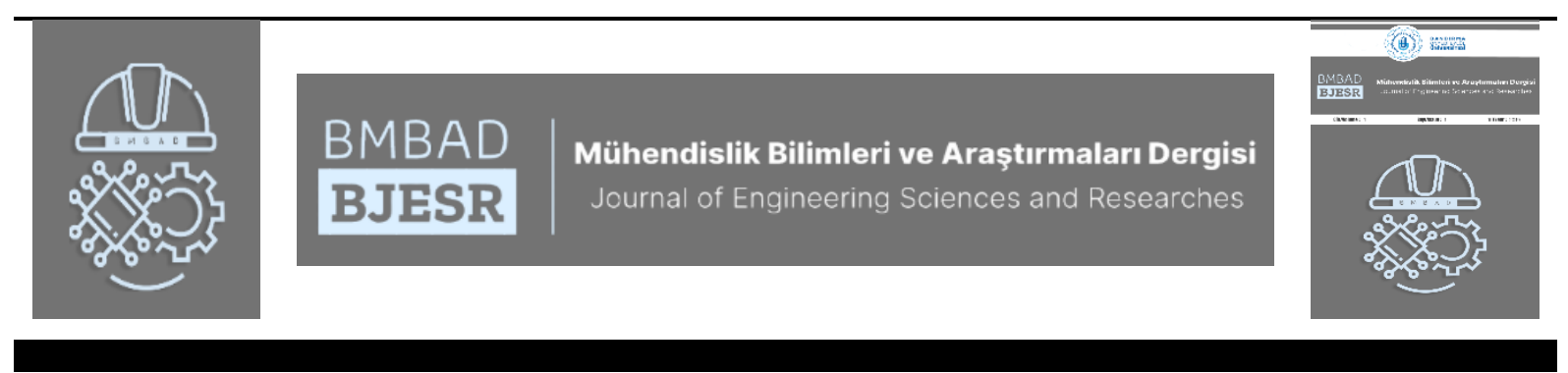

\title{
Görüntii İkileştirme için Global Eşikleme Yöntemleri Üzerine Bir İnceleme
}

\section{A Review on Global Thresholding Methods for Image Binarization}

\begin{abstract}
Abdullah Elen
Bandırma Onyedi Eylül Üniversitesi, Mühendislik ve Doğa Bilimleri Fakültesi, Yazılım Mühendisliği Bölümü, Bandırma, Balıkesir/TÜRKIYE
\end{abstract}

aelen@bandirma.edu.tr

Araştırma Makalesi/Research Article

\begin{tabular}{l}
\hline ARTICLE INFO \\
Article history \\
Received:2 September 2020 \\
Accepted:14 September 2020
\end{tabular}

Accepted:14 September 2020

Keywords:

Image Processing, Global Thresholding, Binarization

\section{ABSTRACT}

Thresholding is one of the most essential issues in image processing. Although this technique is commonly used for segmentation of regions with different homogeneity in grayscale images, and it is also preferred for segmentation of colored images. In this study, 14 different algorithms capable of global thresholding on gray-scale images were categorized and examined in detail as cluster-based, entropy-based, shape-based and feature similarity-based. To test the performance of the algorithms, a dataset consisting of 15 different images was prepared. For these images, the threshold value was determined manually by four experts and reference binary images were obtained by calculating mean value. Accordingly, the binary images produced by each algorithm were examined with the similarity rates to the reference images using the Jaccard Index method. In experimental studies, it was seen that the highest score according to the average similarity ratio obtained belongs to the IsoData and Otsu algorithms with approximately $95 \%$.

(C) 2020 Bandirma Onyedi Eylul University, Faculty of Engineering and Natural Science. Published by Dergi Park. All rights reserved.

\section{MAKALE BİLGISI $\quad$ ÖZET}

Makale Tarihleri

Gönderim: 2 Eylül 2020

Kabul: 14 Eylül 2020

Görüntü işlemede en temel konulardan biri eşikleme yöntemidir. Bu yöntem, yaygın olarak gri tonlamalı görüntülerdeki farklı homojenliğe sahip bölgelerin bölütlenmesinde kullanılmakla birlikte renkli görüntülerin bölütlenmesi için de tercih edilir. Bu çalışmada, gri ölçekli görüntüler üzerinde global eşikleme yapabilen 14 farklı algoritma; kümeleme tabanlı, entropi tabanlı, şekil tabanlı ve öznitelik benzerliğine dayalı olmak üzere kategorize edilmiş ve detaylı olarak incelenmiştir. Algoritmaların performansını test etmek için 15 farklı görüntüden oluşan bir veri seti hazırlanmıştır. Bu görüntüler için dört uzman tarafından el yordamıyla eşik değeri belirlenmiş ve ortalama değeri alınarak referans ikili görüntüler elde edilmiştir. Buna göre her bir algoritmanın ürettiği ikili görüntüler, Jaccard Index yöntemiyle referans görüntülere olan benzerlik oranları incelenmiştir. Deneysel çalışmalarda, elde edilen benzerlik oranı ortalamasına göre en yüksek skor yaklaşık \%95 ile IsoData ve Otsu algoritmalarına ait olduğu görülmüştür.

2020 Bandırma Onyedi Eylül Üniversitesi, Mühendislik ve Dağa Bilimleri Fakültesi. Dergi Park tarafından yayınlanmaktadır. Tüm Hakları Saklıdır. 


\section{GIRIȘ}

Eşikleme, görüntü bölütleme amacı için kullanılan en önemli yaklaşımlardan birisidir [1] ve birçok görüntü analizi uygulamasında ön işlem olarak tercih edilir. Örneğin medikal görüntü işleme, belge görüntülerinin analizi, doku analizi, nesne algılama, parmak izi ve yüz tanıma, haritalar, çizimler vb. birçok alanda problemlerin çözümü için temel yapı niteliğindedir [2,3]. Burada amaç, uygun bir eşik değeri kullanarak görüntü içerisindeki nesneleri arka plandan ayırmaktır [4]. Global algoritmalar, eşik değerini belirlemek için tüm görüntü bilgilerini kullanır ve görüntüdeki tüm piksellere eşit olarak uygulanır [5]. Otomatik eşiklerin temel avantajı, görüntü değişimlerini hesaba katmaları ve kullanıcı önyargısız birçok görüntüyü toplu işlenmesini kolaylaştırmasıdır. Eşik, verilerin karmaşıklığını azaltan, tanıma ve sınıflandırma vb. süreçlerde işlem kolaylığı sağlayan ikili (Binary) bir görüntü üretilmesini mümkün k1lar. Eşikleme, görüntünün birden fazla özelliğini kullanarak bölütleme gerçekleştiren yöntemlere kıyasla daha hızlı sonuç verir [6]. Optimal eşik değerinin tespit edilmesinde, yaygın olarak görüntüdeki gri seviye dağılımlarını gösteren bir histogramdan faydalanılır [7]. Bu durumda nesneleri arka plandan ayırmak için en kolay yol, histogramdan göreceli olarak belirlenen bir $T$ eşik değeri ile görüntüdeki piksel değerlerini karşılaştırmak olacaktır. Başka bir ifadeyle, bir görüntü histogramındaki piksellerin yoğunluk seviyelerine bağlı olarak kümelere ayırma işlemini gerçekleştirmektir. Bu karşılaştırma işlemi (Eşitlik 1) sonucunda 0 ve 1 'den oluşan ikili (Binary) görüntü elde edilir. Buna göre, görüntüdeki herhangi bir $(i, j)$ pikseli için; $f(i, j)>T$ ise nesneye ait bir piksel, $f(i, j) \leq T$ ise arka plana ait bir piksel olacaktır.

$f^{T}(i, j)=\left\{\begin{array}{cc}255, & \text { ĕger } f(i, j)>T(i, j) \text { ise } \\ 0, & \text { değilse }\end{array}\right.$

Burada $T$ değerinin doğru seçilmesi kritik önem taşımaktadır [8]. Eğer $T$ değeri olması gereken daha fazla ya da az olursa görüntünün içerdiği anlamlı bilgi ciddi miktarda zarar görecektir. Bunun sonucu olarak, elde edilen ikili sonuç görüntüsü bir problemin çözümü için geliştirilen sistemin başarısını olumsuz etkileyecektir. Buna ilaveten, az sayıda görüntü için ideal eşik değeri deneme yoluyla bulunabilse de çok sayıda görüntü için bu zaman açısından mümkün olmayabilir. Bu nedenle, dijital görüntülerde eşik değerini otomatik olarak hesaplayan bir algoritmaya her zaman ihtiyaç duyulmuştur [9]. Uzun tarihinin ve geniş uygulamalarının yanı sıra, daha iyi binarizasyon algoritmaları geliştirmenin dışında, nitel ve nicel olarak değerlendirmesi ve bunların karşılaştırması aktif bir araştırma alanı olmaya devam etmektedir. Ayrıca görüntü analizinin geliştirilmesi açısından da son derece önemlidir.

\section{GLOBAL EŞIKLEME YÖNTEMLERİ}

Bugüne kadar gri seviyeli dijital görüntüler için farklı kategorilerde birçok otomatik global eşikleme yöntemi önerilmiştir. Bunlar başlıca kümeleme tabanlı, entropi tabanlı, şekil tabanlı, öznitelik benzerliğine dayalı eşikleme algoritmaları olarak kullanılan bilgi türlerine göre kategorize edilmektedir [10]. Bu çalışmada ele alınan eşikleme yöntemlerinin matematiksel notasyonları ve çalışma prensipleri dört farklı alt başlık altında toplanmıştır. Eşikleme algoritmaların matematiksel eşitliklerinden bahsederken notasyonları şu şekilde kullanacağız; $\forall g \in\{0,1, \ldots, G\}$ için burada $G$ görüntüdeki maksimum gri seviye (parlaklık) değerini temsil etmektedir. Histogram $h(g)$, olasılık kütle fonksiyonu $p(g)$, kümülatif olasılık fonksiyonu $P(g)=\sum_{g=0}^{G} p(g)$, eşikleme değeri $T$ ile gösterilmektedir. Ön plan (nesne) ve arka plan olasılık kütle fonksiyonları sırasıyla $p_{f}(g)$ ve $p_{b}(g)$ olarak ifade edilir ve bunlara ait olasılıklar aşağıdaki gibi hesaplanır;

$$
\begin{aligned}
& P_{b}(T)=P_{b}=\sum_{g=0}^{T} p(g) \\
& P_{f}(T)=P_{f}=\sum_{g=T+1}^{G} p(g)
\end{aligned}
$$

Bu çalışmada, karşılaştırmak için kullandığımız bazı algoritmalar entropi tabanlıdır ve genel olarak Shannon'un entropisi üzerine inşa edilmiştir. Eşikleme değerine bağlı olarak, Shannon'un entropisinin ön plan ve arka plan hesaplamaları şu şekildedir;

$$
\begin{aligned}
& H_{b}(T)=-\sum_{g=0}^{T} p_{b}(g) \log p_{b}(g) \\
& H_{f}(T)=-\sum_{g=T+1}^{G} p_{f}(g) \log p_{f}(g)
\end{aligned}
$$

Bu iki ifadenin toplamı ise; $H(T)=H_{b}(T)+H_{f}(T)$ şeklinde hesaplanır. Bulanık ölçümlerde ise ön plan ve arka plan fonksiyonları sırasıyla $m_{f}(g)$ ve $m_{b}(g)$ olarak ifade edilir ve aşağıda gösterildiği gibi hesaplanır;

$$
\begin{aligned}
& \mu_{b}(T)=\sum_{g=0}^{T} g p(g) \\
& \sigma_{b}^{2}(T)=\sum_{g=0}^{T}\left[g-\mu_{b}(T)\right]^{2} p(g) \\
& \mu_{f}(T)=\sum_{g=T+1}^{G} g p(g)
\end{aligned}
$$


$\sigma_{f}^{2}(T)=\sum_{g=T+1}^{G}\left[g-\mu_{f}(T)\right]^{2} p(g)$

\subsection{Kümeleme Tabanlı Eşikleme}

Kümeleme tabanlı otomatik eşikleme yöntemlerinden en iyi bilineni Otsu [11]'dur. Bu metot kullanılırken görüntünün arka plan ve ön plan olmak üzere iki renk sınıfından oluştuğu varsayımı yapılır. Bu iki sınıfı ayıran optimum eşik değerini $(T)$ bulmak için sınıf içi varyans değeri minimum olacak şekilde hesaplanır;

$\sigma_{W}^{2}(T)=p_{f}(T) \sigma_{f}^{2}(T)+p_{b}(T) \sigma_{b}^{2}(T)$

Burada $p_{f}$ ve $p_{b}, T$ eşik değeri ile ayrılan iki sınıfın kütle olasılıklarını temsil eder. $\sigma_{W}^{2}(T)$ ise bu iki sınıfın varyansıdır. Otsu, sınıf içi varyansı en aza indirmenin, sınıflar arası varyansı maksimuma çıkarmakla aynı olduğunu gösterir. Bu nedenle daha basit olmasından dolayı kullanmak için çok daha iyi bir yaklaşımdır.

$\sigma_{B}^{2}(T)=\sigma^{2}-\sigma_{W}^{2}(T)$

$\sigma_{b}^{2}(T)=p_{f}(T) p_{b}(T)\left[\mu_{f}(T)-\mu_{b}(T)\right]^{2}$

Burada $\sigma_{B}^{2}(T)$ sınıflar arası varyansını, $\mu_{f}$ ve $\mu_{b}$ ise sınıf ortalamalarını temsil etmektedir. Doyle [12] tarafindan önerilen P-tile (Percentile) algoritması gri seviye histogramı tabanlı en eski eşikleme yöntemlerinden biridir. Bir görüntüdeki nesnelerin arka plandan daha parlak olduğunu ve nesnelerin görüntü üzerinde sabit bir alan yüzdesini işgal ettiğini varsayar. Bu sabit alan yüzdesi de $K \%$ olarak bilinir. Eşik değeri $(T)$, çoğunlukla gri seviyenin nesneye en az $K \%$ oranında eşleştirilmesine karşlık gelen gri seviye olarak tanımlanır. $\mathrm{Bu}$ oran otomatik işlemlerde genelde $K=0.5$ olarak belirlenir. P-tile yönteminin algoritması Eşitlik 14'teki gibidir;

$P=\sum_{g=0}^{G} h(g)$

$T=\underset{g}{\operatorname{argmin}}\left\{\frac{1}{P} \sum_{g=0}^{G} p(g) \geq K\right\}$

Burada $P$ histogramdaki kümülatif olasılık toplam fonksiyonunu, $T$ ise optimum eşik değerini temsil etmektedir. Simple Image Statistic (SIS), Kittler ve Illingworth [13] tarafından geliştirilen otomatik iki seviyeli bir eşik belirleme tekniğidir. Bu yöntemde, gri tonlamalı bir görüntüdeki gradyan büyüklüğüyle ağırlıklandırılmıs piksel parlaklığına dayalı olarak optimum global eşik değeri Eşitlik 16'da gösterildiği gibi tahmin edilir.

$e(i, j)=|\nabla p|=\max \{|l(i-1, j)-l(i+1, j)|,|l(i, j-1)-l(i, j+1)|\}$

$T_{\text {opt }}=\frac{\sum_{i=1}^{m} \sum_{j=1}^{n}|l(i, j) \times e(i, j)|}{\sum_{i=1}^{m} \sum_{j=1}^{n}|e(i, j)|}$

Burada $l(i, j)$ ve $e(i, j)$ sırasıyla görüntüdeki parlaklığı ve gradyan büyüklüğünü temsil etmektedir. Piksel cinsinden görüntü boyutları ise $m$ ve $n$ olarak gösterilmektedir. Ridler ve Calvard [14], IsoData olarak adlandırılan otomatik olarak global eşik değerini belirleyen iteratif bir algoritma geliştirmişlerdir. Bu algoritmada ilk olarak, histogram $T_{0}$ gibi bir başlangıç eşik değeri belirlenerek iki kısma ayrılır. Yani $T_{0}$ histogramdaki piksel sayısı sıfırdan farklı en küçük parlaklık değeridir.

$T_{\text {opt }}=\lim _{n \rightarrow \infty} \frac{\mu_{f}\left(T_{n}\right)+\mu_{b}\left(T_{n}\right)}{2}$

$\mu_{f}\left(T_{n}\right)=\sum_{g=0}^{T_{n}} g p(g)$

$\mu_{b}\left(T_{n}\right)=\sum_{g=T_{n}+1}^{G} g p(g)$

Daha sonra $T_{0}$ eşik değeri referans alınarak, görüntü üzerindeki nesnelere ve arka plana ait gri seviyeli değerlerin ortalamaları sirasıyla $\mu_{f}$ ve $\mu_{b}$ olarak bulunur. Bir sonraki adımda ise $\mu_{f}$ ve $\mu_{b}$ değerlerinin aritmetik ortalaması alınarak yeni bir $T_{1}$ eşik değeri hesaplanır. Süreç, eşik değeri artık değişmeyene kadar $\left(T_{n}\right)$ yeni eşiğe bağlı olarak tekrarlanır. Bir diğeri ise Mean [15] yöntemidir. Bu metot gri seviye dağılımının ortalamasını eşik olarak seçer. Bununla birlikte, diğer bazı yöntemler bunu ilk tahmin eşiği olarak kullanır.

$T=\sum_{g=0}^{G} g p(g)$ 


\subsection{Entropi Tabanlı Eşikleme}

Entropi kavramı ilk kez Shannon tarafından bilgisayar bilimlerinde sinyal işleme ve veri iletişiminde kullanılmıştır. Enformasyon teorisinde bilginin ortalama miktarını ölçmek için de entropiden yararlanılır. Görüntü analizinde entropi, rastgele bir değişkenin minimum tanımlayıcı karmaşıklığını ölçmek için kullanılır. Bu yönü ile görüntü işleme alanında da oldukça tercih edilen bir kavramdır. Kapur ve ark. [16], MaxEntropy olarak bilinen algoritmasında, görüntü histogramındaki gri düzeylerin dağılımının entropisini hesaplayabilmek için Shannon'un konseptini kullanmıştır. Görüntüdeki nesneler ve arka plan iki farklı sinyal kaynağı olarak düşünülürse, bu iki sınıfa ait entropilerinin toplamı maksimum seviyeye ulaştığı durumda optimum eşik değeri bulunmuş olur.

$$
\begin{aligned}
& T_{o p t}=\operatorname{argmax}\left[H_{f}(T)+H_{b}(T)\right] \\
& H_{f}(T)=-\sum_{g=0}^{T} \frac{p(g)}{p(T)} \log _{e} \frac{p(g)}{p(T)} \\
& H_{b}(T)=-\sum_{g=T+1}^{G} \frac{p(g)}{p(T)} \log _{e} \frac{p(g)}{p(T)}
\end{aligned}
$$

Sahoo ve ark. [17], Renyi entropisini kullanarak görüntü histogramında $T_{1}, T_{2}$ ve $T_{3}$ olmak üzere üç farklı eşik değeri bulunur. Bu eşik değerleri $\rho$ parametresinin $\rho>1,0<\rho<1$ ve $\rho=1$ durumları esas alınarak Eşitlik 24 ve 25 'te gösterildiği gibi hesaplanır.

$$
\begin{aligned}
& H_{f}^{\rho}=\frac{1}{1-\rho} \ln \left\{\sum_{g=0}^{T}\left[\frac{p(g)}{p(T)}\right]^{\rho}\right\} \\
& H_{b}^{p}=\frac{1}{1-p} \ln \left\{\sum_{g=T+1}^{G}\left[\frac{p(g)}{1-p(T)}\right]^{p}\right\}
\end{aligned}
$$

Optimum eşik değeri ise nesnelere ve arka plana ait gri seviyelerin Renyi entropi toplamlarını maksimize edilerek Eşitlik 26'da gösterildiği gibi hesaplanır.

$$
\begin{aligned}
& T_{\text {opt }}=T_{[1]}\left[P_{T_{[1]}}+\frac{1}{4} w B_{1}\right]+T_{[2]} \frac{1}{4} w B_{2}+T_{[3]}\left[1-P_{T_{[3]}}+\frac{1}{4} w B_{3}\right] \\
& P\left[T_{[k]}\right]=\sum_{g=0}^{T_{[k]}} p(g), \forall k \in\{1,2,3\} \\
& x=\left|T_{[1]}-T_{[2]}\right|, y=\left|T_{[1]}-T_{[2]}\right| \\
& B_{1}, B_{2}, B_{3}= \begin{cases}(1,2,1) & \text { if } x \leq 5 \text { and } y \leq 5 \text { or } x>5 \text { and } y>5 \\
(0,1,3) & \text { if } x \leq 5 \text { and } y>5 \\
(3,1,0) & \text { if } x>5 \text { and } y \leq 5\end{cases}
\end{aligned}
$$

Li ve ark. $[18,19]$, ikili ve orijinal görüntü arasındaki çapraz-entropinin minimize edilebilmesi için kullanılan yinelemeli bir yöntem önermişlerdir. Bu, görüntüler arasındaki veri tutarlılı̆ının bir ölçüsü olarak yorumlanır ve optimal eşiklemeyi Kullback-Leibler mesafesinin en aza indirilmesi olarak formüle eder. Kullback-Leibler ölçüsü ile gözlemlenen ve yeniden oluşturulan görüntülerin, ön plan ve arka planları aynı ortalama yoğunluğa sahip olduğu kısitlar altında minimize eder.

$$
T_{o p t}=\operatorname{argmin}\left[\sum_{g=0}^{T} g p(g) \log \frac{g}{m_{f}(T)}+\sum_{g=T+1}^{G} g p(g) \log \frac{g}{m_{b}(T)}\right]
$$

Where $\square \sum_{g \leq T} g=\sum_{g \leq T} m_{f}(T) \square$ and $\square \sum_{g \geq T} g=\sum_{g \geq T} m_{b}(T) \square$

Shanbhag [20] tarafından önerilen yöntem, Kapur ve ark. [16] tarafından önerilen maksimum entropi eşikleme tekniğinin farklı bir sürümüdür. Shanbhag eşiklemesi bulanık üyeleri (fuzzy memberships), görüntüdeki gri seviyelerin nesneye veya arka plana ne kadar güçlü bir şekilde ait olduğunun bir göstergesi olarak görür. $\mathrm{Bu}$ yöntemde, bir görüntü iki bulanık kümeye karşlık gelen iki farklı sınıfa ayırılır: $\mu_{f}$ histogramdaki 0 'dan $T$ 'ye kadar olan gri seviyeleri, $\mu_{b}$ ise $T+1$ 'den $G$ 'ye kadar olan gri seviyeleri temsil etmektedir. Üyelik katsayıları, ilgili gri seviyenin bir sınıfa atanmasındaki belirsizlik olarak ölçülür. Açıkçası, yalnızca minimum ve maksimum olası gri düzeylerine sahip pikseller, maksimum üyelik katsayısına göre $\mu_{f}$ veya $\mu_{b}$ 'ye atanacaktır. Bu da yüksek değerlikli gri seviyelerin herhangi bir belirsizlik olmadan kendi sınıflarına nasıl atanacağını açıklar. Bununla birlikte, $T$ ve $T+1$ eşik değerleri arasında farazi bir $T^{\prime}$ olduğu varsayılır. Bu nedenle, $T^{\prime}$ gri değerine sahip piksel değerleri 0.5 'lik üyelik katsayısına sahip olacaktır. Bir sınıfa göre herhangi bir gri seviyenin üyelik katsayısı, bu gri seviyeye sahip olan piksellerin bulunma olasıllı̆ı ile de artar. 
$\mu_{f}(T-i)=0.5+\frac{P(T)+\ldots+P(T-1-i)+P(T-i)}{2 P(T)}$
$\mu_{b}(T+i)=0.5+\frac{P(T+1)+\ldots+P(T-1+i)+P(T-i)}{2(1-P(T))}$

Sonuç olarak, bir sınıf içindeki eşikten daha büyük bir piksel yoğunluğu daha yüksek üyelik olarak belirlenir. Böylece, arka plan ve nesnelerin entropileri hesaplanır ve optimum eşik değeri, iki entropi arasındaki farkı minimize eden $T_{\text {opt }}$ olarak Eşitlik 34'teki gibi belirlenir.

$T_{\text {opt }}=\operatorname{argmin}\left\{\left|H_{f}(T)-H_{b}(T)\right|\right\}$

$H_{f}(T)=-\sum_{g=0}^{T} \frac{P(g)}{P(T)} \log \left[\mu_{f}(g)\right]$

$H_{b}(T)=-\sum_{g=T+1}^{G} \frac{P(g)}{1-P(T)} \log \left[\mu_{b}(g)\right]$

Yen ve ark. [21], Renyi entropisindeki güç parametresini $\rho=2$ olarak belirleyerek, optimum eşik değerini maksimize eden bir entropik korelasyon yöntemi önermişlerdir. Buna göre görüntüdeki optimal eşik değeri Eşitlik 37'deki gibi hesaplanır.

$$
\begin{aligned}
& T_{o p t}=\operatorname{argmax}\left\{C_{b}(T)+C_{f}(T)\right\} \\
& C_{b}(T)=-\log \left\{\sum_{g=0}^{T}\left[\frac{p(g)}{p(T)}\right]^{2}\right\} \\
& C_{f}(T)=-\log \left\{\sum_{g=T+1}^{G}\left[\frac{p(g)}{1-p(T)}\right]^{2}\right\}
\end{aligned}
$$

\section{3. Şekil Tabanlı Eşikleme}

$\mathrm{Bu}$ yöntem kategorisindeki algoritmalar, görüntü histogramı üzerinde geometrik şekil benzerliği, eğri analizi, referans tepe noktaları, vadiler vb. biçimde şekil özelliklerine göre eşik değeri seçerler. Bunlardan en çok bilineni Zack'in Triangle [22] algoritmasıdır. Bu yöntemde, gri seviyeli bir görüntü histogramında maksimum tepe değeri $y_{\max }$ ve minimum tepe değeri $y_{\min }$ bulunur. Aynı zamanda bu tepe değerlerine karşılık gelen $x_{\min }$ ve $x_{\max }$ gri seviye değerleri de tespit edilmiş olur. Daha sonra bu noktaların arası Şekil 1'de gösterildiği gibi bir çizgi ile birleştirilerek $d: a x+b y+c=0$ doğrusu elde edilir.

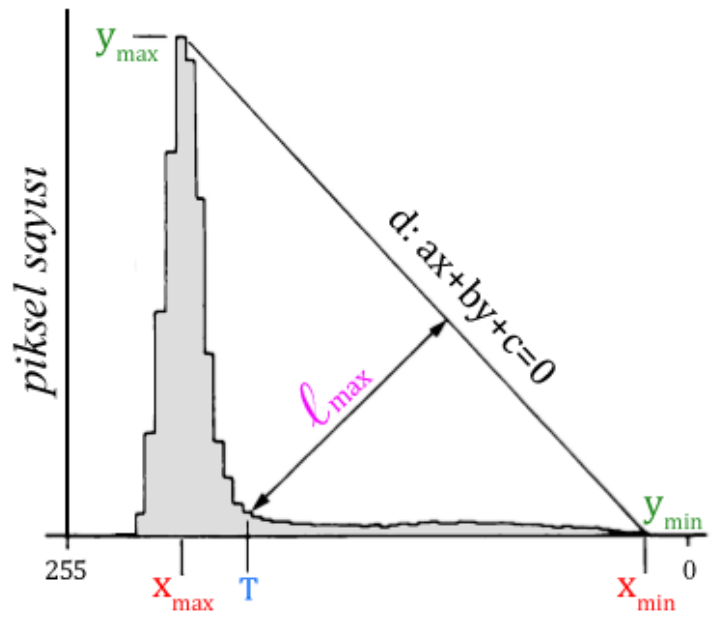

Şekil 1. Zack'in üçgen yöntemi.

$$
\begin{aligned}
& x_{\text {min }}=\underset{g}{\operatorname{argmin}}\left\{\sum_{g=0}^{G} p(g)>0\right\}, y_{\text {min }}=h\left(x_{\text {min }}\right) \\
& x_{\text {max }}=\underset{g}{\operatorname{argmax}}\left\{\sum_{g=0}^{G} p(g)>0\right\}, y_{\text {max }}=h\left(x_{\text {max }}\right)
\end{aligned}
$$

Ancak burada bilinmesi gereken bir şey daha vardır. Histogramda $x_{\min }$ değeri sağ tarafta olabileceği gibi sol tarafta da olabilir. Bu durumda $x_{\min }$ ile $x_{\max }$ değerleri yer değiştirilir. Ayrıca tüm gri seviyeler eşit durumda $x_{\min }=x_{\max }$ olursa optimum $T$ değeri $x_{\min }$ veya $x_{\max }$ 'tan biri olarak belirlenir. Daha sonra bu bilgileri kullanarak, iki noktası bilinen $d$ doğrusunun denklemini aşağıdaki gibi elde edilir. 
$d:\left(x-x_{\max }\right)\left(y_{\max }-y_{\min }\right)-\left(y-y_{\max }\right)\left(x_{\max }-x_{\min }\right)$

Bu eşitliği $d: a x+b y+c=0$ olduğunu varsayarsak, optimum eşik değeri aşağıdaki formülde gösterildiği gibi hesaplanır. Yani $l$ uzunluğunun maksimum olduğu $\left(l_{\max }\right)$ yerdeki $x_{n}$ değeridir.

$T_{o p t}=\underset{n}{\operatorname{argmax}}\left\{n \mid n \in\left\{x_{\min }, \ldots, x_{\max }\right\}, \frac{|a n+b h(n)+c|}{\sqrt{a^{2}+b^{2}}}\right\}$

Bu teknik, histogramda nesneye ait piksellerin zayıf bir tepe meydana getirdiği durumlarda etkilidir. Prewit ve ark. [23], kan hücrelerinin analizi için yaptıkları çalışmada periferik kan yayma görüntülerinin gri seviyeli histogramı üzerinden lökosit hücrelerinin nükleus ve sitoplazmalarını segmente etmek istemişlerdir. Bunun için Intermodes olarak bilinen çift modlu (bimodal) bir eşikleme yöntemi önermişlerdir. Bu yöntem, histogramın iki modlu bir dağılımı olduğunu varsayar ve iki yerel maksimumu lokalize etmeye çalışır. Bu yerel maksimumlar bulunduğunda, yoğunlukları arasındaki ortalama optimum eşik değeri olarak belirlenir. Geniş ve düz bir vadiye veya oldukça düzensiz yapıda zirvelere sahip olan histogramlar bu yöntem için uygun değildir.

\section{4. Öznitelik Benzerliğine Dayalı Eşikleme}

Bu kategorideki algoritmalar, orijinal görüntü ile ikili görüntü arasında bazı özellik kalitesi veya benzerlik ölçüsüne dayalı eşik değerini belirler. Bu nitelikler, kenar eşleştirmesi, şekil kompaktlığı, moment, bağlantı, doku veya segmente edilmiş nesnelerin kararlılığı olarak söylenebilir. Tsai [24], Gri seviyeli bir görüntüyü, ideal bir ikili görüntünün bulanık sürümü olarak görür. Optimum eşikleme, ilk üç gri seviye momentinin, ikili görüntünün ilk üç momentine denk gelecek şekilde Eşitlik 46'daki gibi oluşturulur. Gri seviyeli momentler $m_{k}$ olarak, binary görüntü momentleri ise $b_{k}$ olarak tanımlanmıştır.

$$
\begin{aligned}
& m_{k}=\sum_{g=0}^{G} p(g) g^{k} \\
& b_{k}=P_{f} m_{f}^{k}+P_{b} m_{b}^{k} \\
& T_{o p t}=\arg \text { e qual }\left[m_{1}=b_{1}(T), m_{2}=b_{2}(T), m_{3}=b_{3}(T)\right]
\end{aligned}
$$

Huang ve ark. [25], gri ölçekli görüntülerin histogram bilgilerini kullanarak, bulanık entropi-tabanlı (fuzzy entropybased) bir eşikleme yöntemi geliştirmişlerdir. Bu yöntem, gri ölçekli görüntü ile ikili görüntü arasındaki mesafeyi ölçerek bir bulanıklık indeksi oluşturur. Görüntü $m_{f}(I)$ dizisi olarak temsil edilir ve burada $0 \leq m_{f}(I) \leq 1$ olarak, ön plana ait olan bulanık ölçüyü temsil eder. Her bir görüntü pikseli için bulanık üyelik değeri göz önüne alındığında, Shannon'un veya Yager'in entropi fonksiyonuyla görüntünün tümü için bir bulanıklık indeksi elde edilebilir. Optimal eşik, sinıf (ön ve arka plan) ortalama (mean) $m_{f}(T), m_{b}(T)$ ve üyelik fonksiyonları $\mu_{f}[I(i, j), T], \mu_{b}[I(i, j), T]$ açısından tanımlanan bulanıklık indeksi minimize ederek, Eşitlik 47 'de gösterildiği gibi hesaplanır $[10,26]$.

$$
\begin{aligned}
& T_{o p t}=\operatorname{argmin}\left\{-\frac{1}{N^{2} \log 2} \sum_{g=0}^{G}\left(\begin{array}{l}
{\left[\mu_{f}(g, T) \log \left(\mu_{f}(g, T)\right)\right]+} \\
{\left[1-\mu_{f}(g, T)\right] \log \left[1-\mu_{f}(g, T)\right] p(g)}
\end{array}\right)\right\} \\
& \mu_{f}[I(i, j), T]=\frac{G}{G+\left|I(i, j)-m_{f}(T)\right|}
\end{aligned}
$$

\section{DENEYSEL ÇALIŞMALAR}

Deneylerde kullanılmak üzere her birinde beş görüntü bulunan üç bölüm halinde veri seti (Şekil 2, Şekil 3 ve Şekil 4) hazırlanmıştır. Bunlar görüntü işleme çalışmalarında sıklıkla kullanılan (iyi bilinen) görüntüler, biyolojik, coğrafi, kültürel ve diğer çeşitli türlerde olan görüntülerdir. Veri setleri yalnızca fiziksel nedenlerden dolayı (sayfa boyutu ve görüntü sayısı) gruplandırılmıştır. Görüntüler $512 \times 512$ piksel boyutlarında ve gri ölçeklidir (8-bit). Deneyde kullanılan tüm görüntüler için dört farklı uzman tarafından uygun bir eşik değeri belirlenmiştir. Bunun sebebi yapılan çalışmayı kişisel önyargıdan uzak tutmaktır. Daha sonra uzman kişiler tarafından belirlenen her bir görüntü için eşik değerlerin ortalaması alınarak hesaplanan referans eşik değerleri $(T)$ olarak belirtilmiştir. Buna göre giriş görüntülerinin $T$ eşik değerine göre ikili görüntüleri elde edilmiştir. Tüm görüntü seti için uzmanlar arasındaki görüş farklılığının ortalama standart sapma değeri 3.87'dir. Görüntü setlerinin verildiği her bir tabloda giriş görüntüsü, $T$ eşik değerine göre oluşturulmuş ikili referans (ground-truth) görüntüsü (GTI) ve giriş görüntüsüne ait histogram grafiği ile buna ait bazı istatistiksel değerler verilmiştir. 


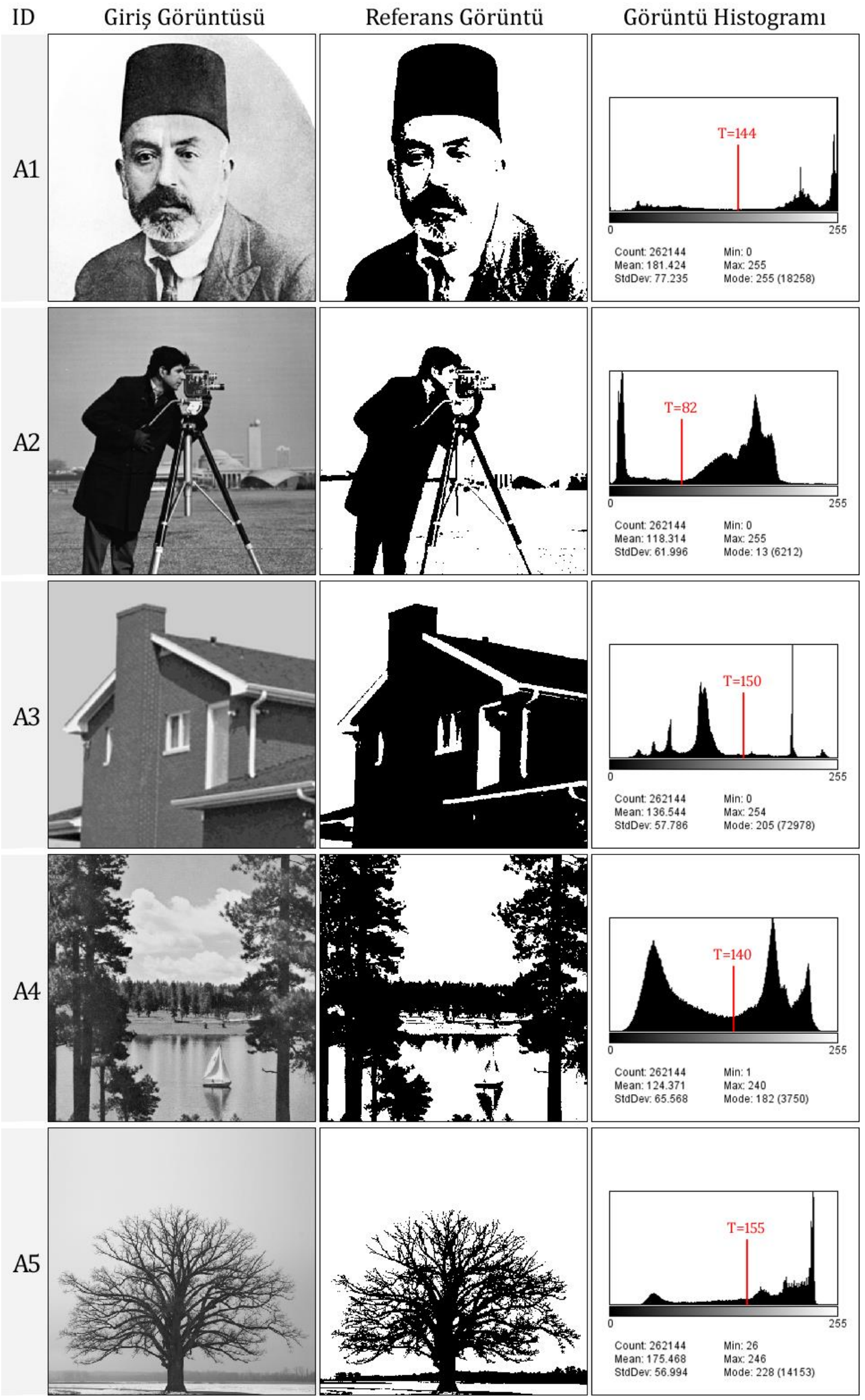

Şekil 2. Veri Seti (Bölüm A) 


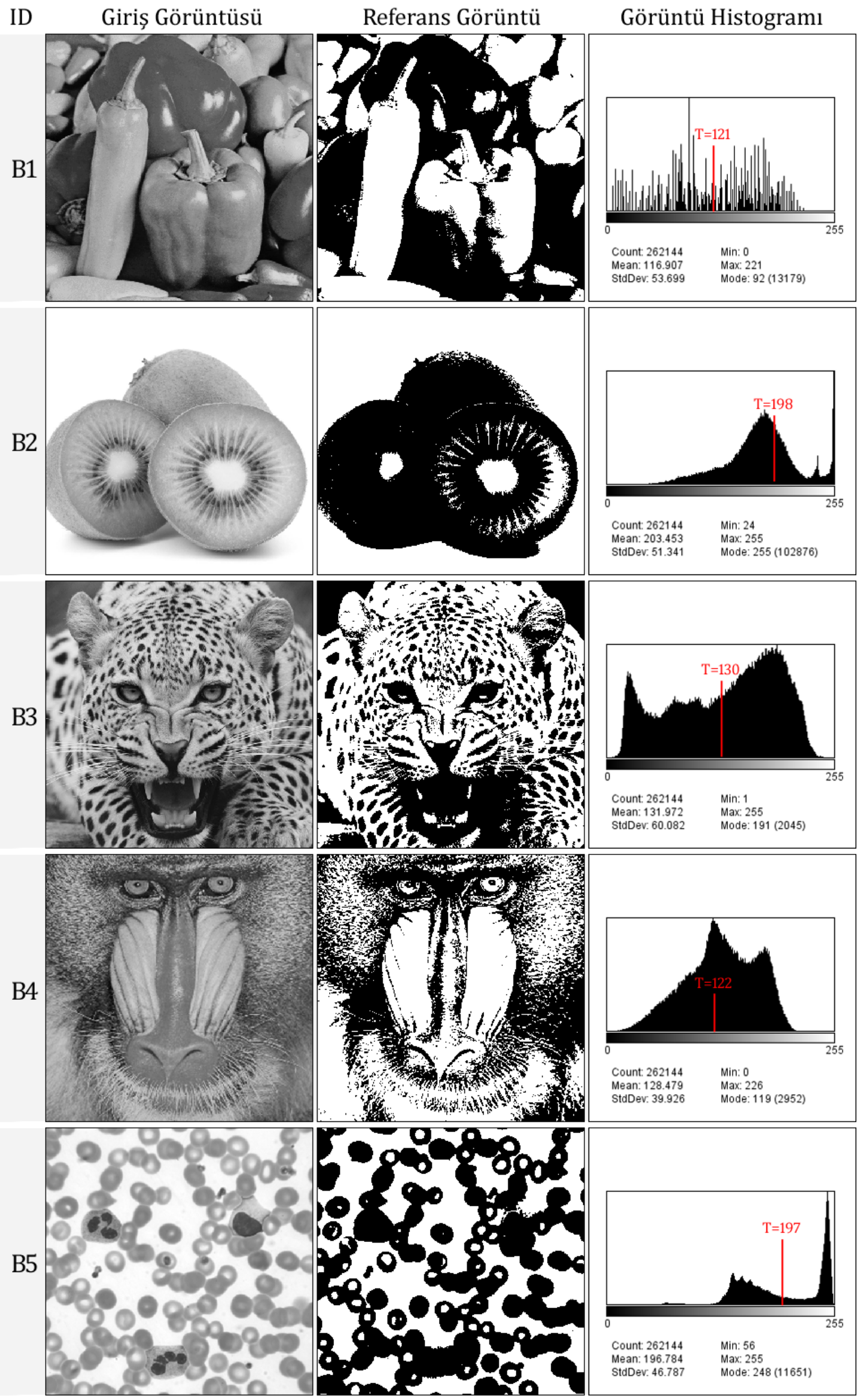

Şekil 3. Veri Seti (Bölüm B) 


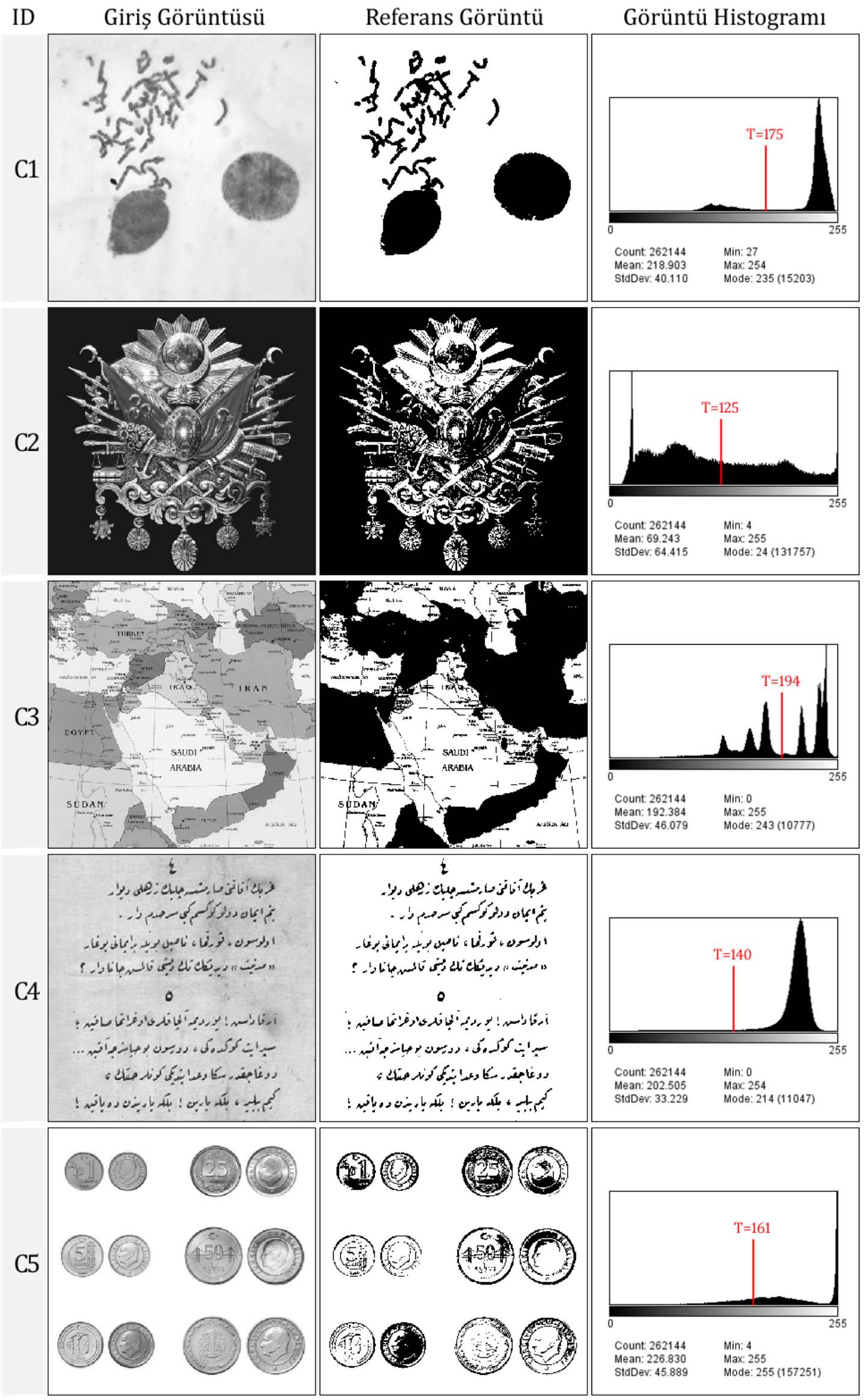

Şekil 4. Veri Seti (Bölüm C) 
Deneysel çalışmalarda, görüntü ikileştirilmesi için 14 farklı global eşikleme algoritması test edilmiştir. Bu algoritmalar; Huang (HG) [25], Intermodes (IM) [23], IsoData (IS) [14], Li (LI) [18, 19], Max entropy (ME) [16], Mean (MN) [15], Moments (MT) [24], Otsu (OT) [11], Percentile (PT) [12], Renyi entropy (RE) [17], Shanbhag (SH) [20], Simple Image Statistics (SI) [13], Zack's triangle (ZT) [22] and Yen (YN) [21] olarak tablo ve grafiklerdeki gösterimleri için kısaltılmıştır. Ayrıca deneylerde referans görüntüsü (Ground-truth) için de (GTI) kısaltması kullanılmıştır. Veri setindeki tüm görüntüler için her bir algoritma tarafından hesaplanan optimum eşik değerleri Tablo 1'de gösterilmektedir.

Tablo 1. Veri setindeki görüntülerin algoritmalara göre optimal ve referans eşik değerleri.

\begin{tabular}{|c|c|c|c|c|c|c|c|c|c|c|c|c|c|c|c|}
\hline \# & GTI & HG & IM & IS & LI & ME & MN & MT & OT & PT & RE & SH & SI & ZT & YN \\
\hline$\overline{\mathrm{A} 1}$ & 144 & 141 & 128 & 148 & 128 & 169 & 181 & 157 & 149 & 214 & 173 & 74 & 133 & 235 & $\overline{188}$ \\
\hline A2 & 82 & 58 & 88 & 88 & 63 & 195 & 118 & 112 & 88 & 142 & 191 & 133 & 112 & 23 & 198 \\
\hline A3 & 150 & 165 & 155 & 148 & 140 & 95 & 136 & 114 & 148 & 111 & 95 & 38 & 124 & 201 & 96 \\
\hline A4 & 140 & 114 & 116 & 124 & 110 & 123 & 124 & 126 & 125 & 123 & 123 & 119 & 112 & 163 & 123 \\
\hline A5 & 155 & 180 & 137 & 142 & 127 & 151 & 175 & 153 & 143 & 197 & 155 & 111 & 135 & 221 & 160 \\
\hline B1 & 121 & 135 & 126 & 117 & 106 & 120 & 116 & 106 & 117 & 117 & 123 & 129 & 115 & 96 & 128 \\
\hline B2 & 198 & 237 & 214 & 205 & 201 & 131 & 203 & 181 & 206 & 199 & 138 & 36 & 160 & 252 & 139 \\
\hline B3 & 130 & 130 & 108 & 120 & 105 & 123 & 131 & 124 & 121 & 143 & 123 & 120 & 126 & 113 & 124 \\
\hline B4 & 122 & 141 & 148 & 126 & 120 & 105 & 128 & 122 & 128 & 129 & 105 & 128 & 123 & 98 & 105 \\
\hline B5 & 197 & 211 & 195 & 198 & 194 & 126 & 196 & 178 & 199 & 193 & 125 & 165 & 188 & 232 & 125 \\
\hline C1 & 175 & 173 & 178 & 182 & 176 & 218 & 218 & 181 & 182 & 234 & 215 & 126 & 192 & 216 & 220 \\
\hline $\mathrm{C} 2$ & 125 & 39 & 106 & 103 & 73 & 145 & 69 & 110 & 104 & 24 & 110 & 15 & 124 & 27 & 102 \\
\hline C3 & 194 & 214 & 204 & 191 & 188 & 118 & 192 & 172 & 192 & 201 & 118 & 160 & 168 & 225 & 118 \\
\hline C4 & 140 & 158 & 133 & 149 & 135 & 168 & 202 & 162 & 150 & 211 & 167 & 63 & 167 & 185 & 168 \\
\hline C5 & 161 & 231 & 219 & 205 & 200 & 119 & 226 & 184 & 206 & 254 & 234 & 7 & 167 & 250 & 249 \\
\hline
\end{tabular}

Her bir algoritma için eşikleme sonucunda elde edilen ikili görüntülerin benzerliklerini hesaplamada Eşitlik 49' da verilen Jaccard Index (Jaccard benzerlik katsayısı) yönteminden faydalanılmıştır. Bu yönteme göre işlemin sonucunda hesaplanan değer 0 ile 1 arasındadır. İşlemin sonucu 1'e ne kadar yakınsa, iki görüntü birbirine o kadar benzerdir.

$J I X=\frac{\left(\sum_{i=0}^{m} \sum_{j=0}^{n} f_{G}(i, j) \wedge f_{T}(i, j)\right)}{\left(\sum_{i=0}^{m} \sum_{j=0}^{n} f_{G}(i, j) \vee f_{T}(i, j)\right)}$

Burada $m$ ve $n$ görüntünün boyutlarını, $f_{G}$ referans görüntüsünü ve $f_{T}$ ise eşikleme sonucunda elde edilen ikili çıkış görüntüsünü temsil etmektedir. Tablo 2'de görüntü setindeki tüm görüntüler için her bir eşikleme algoritmasına göre JIX benzerlikleri verilmiştir. Algoritmaların benzerlik değerlendirmesinde, uzman görüşüne göre eşikleme (GTI) yapılan ikili referans görüntüler esas alınmıştır.

Tablo 2. Jaccard Index yöntemine göre algoritmalar tarafından üretilen ikili görüntülerin benzerlik yüzdeleri.

\begin{tabular}{|c|c|c|c|c|c|c|c|c|c|c|c|c|c|c|}
\hline$\#$ & HG & IM & IS & $\mathbf{L I}$ & ME & MN & MT & OT & PT & $\mathbf{R E}$ & SH & SI & $\mathbf{Z T}$ & YN \\
\hline$\overline{A 1}$ & 99,4 & 97,4 & 99,5 & 97,4 & 96,0 & 93,5 & 98,0 & 99,4 & 72,2 & 95,2 & 85,6 & 98,2 & 46,1 & 91,9 \\
\hline A2 & 96,9 & 99,4 & 99,4 & 97,6 & 1,8 & 87,6 & 91,4 & 99,4 & 68,1 & 2,3 & 75,2 & 91,4 & 91,6 & 1,6 \\
\hline $\mathbf{A 3}$ & 95,2 & 98,8 & 99,2 & 96,9 & 49,2 & 96,0 & 82,7 & 99,2 & 77,4 & 49,2 & 40,3 & 92,3 & 87,0 & 49,5 \\
\hline A4 & 89,8 & 90,6 & 93,6 & 88,2 & 93,2 & 93,6 & 94,4 & 94,0 & 93,2 & 93,2 & 91,8 & 89,0 & 88,0 & 93,2 \\
\hline A5 & 82,2 & 93,6 & 95,0 & 91,1 & 98,1 & 86,3 & 98,9 & 95,3 & 68,8 & 99,6 & 87,8 & 93,1 & 36,3 & 98,4 \\
\hline B1 & 88,3 & 97,7 & 96,5 & 87,6 & 98,7 & 96,1 & 87,6 & 96,5 & 96,5 & 98,5 & 93,3 & 95,3 & 80,0 & 96,0 \\
\hline B2 & 84,4 & 90,1 & 94,8 & 98,1 & 55,7 & 96,3 & 80,1 & 94,1 & 100 & 56,7 & 50,7 & 63,4 & 80,3 & 56,9 \\
\hline B3 & 99,2 & 86,3 & 92,5 & 84,9 & 94,3 & 100 & 95,0 & 93,1 & 89,9 & 94,3 & 92,5 & 96,3 & 88,7 & 95,0 \\
\hline B4 & 70,2 & 60,5 & 94,4 & 94,6 & 77,6 & 90,9 & 98,2 & 90,9 & 89,2 & 77,6 & 90,9 & 100 & 73,2 & 77,6 \\
\hline B5 & 92,8 & 98,1 & 100 & 97,4 & 50,1 & 98,7 & 85,1 & 99,4 & 96,7 & 50,0 & 73,7 & 93,3 & 82,5 & 50,0 \\
\hline C1 & 99,8 & 99,8 & 99,6 & 100 & 96,4 & 96,4 & 99,7 & 99,6 & 63,3 & 96,9 & 92,4 & 98,9 & 96,8 & 96,0 \\
\hline $\mathrm{C2}$ & 45,4 & 82,6 & 80,3 & 58,8 & 82,6 & 56,4 & 85,8 & 81,1 & 20,3 & 85,8 & 19,9 & 98,1 & 42,3 & 79,5 \\
\hline C3 & 91,0 & 97,4 & 99,1 & 98,3 & 54,0 & 99,3 & 74,7 & 99,3 & 98,2 & 54,0 & 67,8 & 71,4 & 71,4 & 54,0 \\
\hline C4 & 98,9 & 99,5 & 99,5 & 99,6 & 97,9 & 82,7 & 98,5 & 99,4 & 56,8 & 98,0 & 96,0 & 98,0 & 94,9 & 97,9 \\
\hline $\mathrm{C5}$ & 77,4 & 79,5 & 83,3 & 85,0 & 91,4 & 78,1 & 91,5 & 82,9 & 72,1 & 77,1 & 88,1 & 98,2 & 75,6 & 75,8 \\
\hline Mean & 87,4 & 91,4 & 95,1 & 91,7 & 75,8 & 90,1 & 90,8 & 94,9 & 77,5 & 75,2 & 76,4 & 91,8 & 75,6 & 74,2 \\
\hline
\end{tabular}

Tablonun son satırında ise veri setindeki tüm görüntülerin ortalama benzerlik oranı hesaplanmıştır. Buna ek olarak, tablodaki her bir görüntü için en iyi benzerlik oranına sahip metotlar kırmızı ile renklendirilmiştir. Ortalama benzerlik oranları mukayese edildiğinde, en yüksek skor yaklaşık \%95 ile IsoData ve Otsu algoritmalarına ait olduğu görülmektedir. Eşikleme algoritmalarının yedi tanesi referans görüntüler ile \%90'1n üzerinde bir benzerliğe 
sahiptir. Yen'in yöntemi ise $\% 74,2^{\prime}$ lik skorla benzerlikte en son sırada yer almaktadır. Şekil 5'te eşikleme algoritmalarının GTI'ya göre ortalama benzerlik grafiği gösterilmektedir.

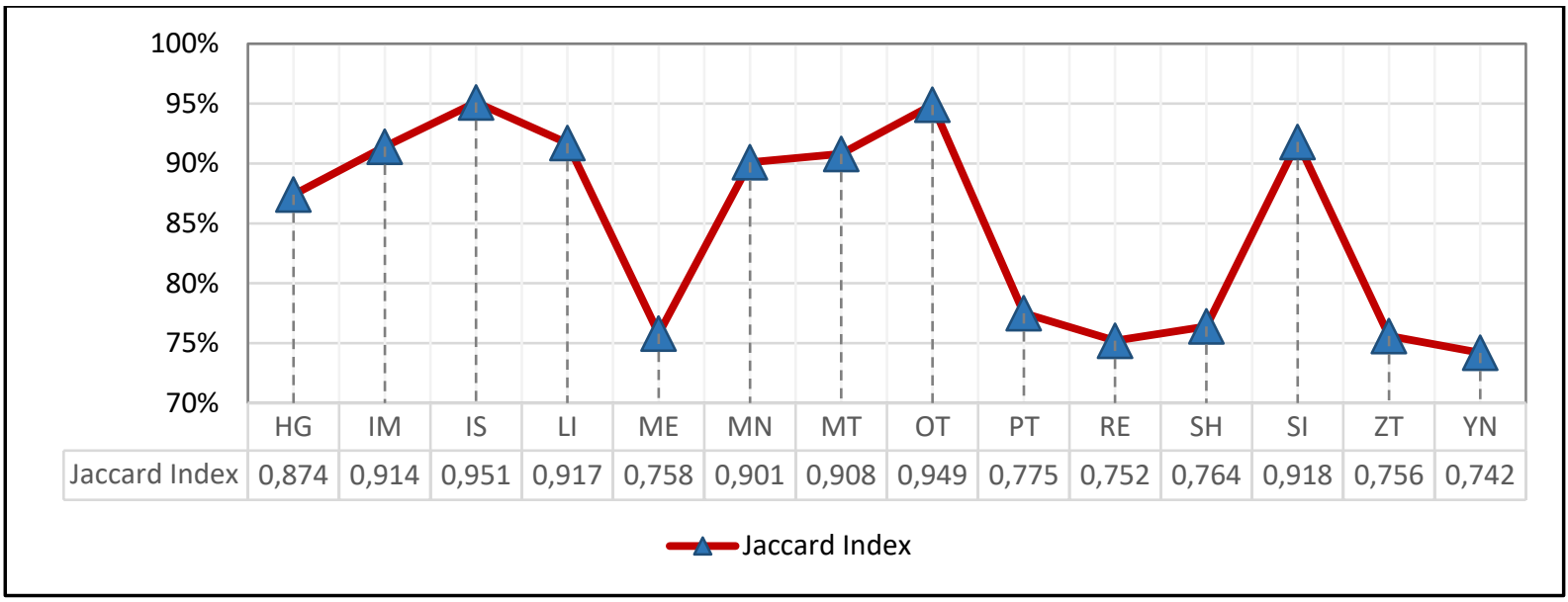

Şekil 5. Jaccard Index yöntemine göre algoritmaların GTI ile benzerlikleri.

\section{SONUÇLAR}

$\mathrm{Bu}$ çalışmada gri-ölçekli görüntülerden oluşan bir veri seti için optimal eşik değerlerinin belirlenmesinde global eşikleme algoritmaları kullanılmıştır. Bu algoritmalar, herhangi bir parametreye ihtiyaç duymaksızın otonom olarak işlemleri gerçekleştirebilmektedir. Deneysel çalışmalarda kullanılan görüntü örneklerinin referans eşik değerleri hesaplanırken, kişisel önyargılara takılmamak için dört farklı uzman tarafından eşik değeri belirlenmesi istenmiştir. Bunun sonucunda da her bir örnek görüntü için uzmanların belirlediği eşik değerlerinin ortalaması alınarak, ikili referans görüntüleri oluşturulmuştur. Veri setindeki tüm görüntüler için uzman görüş farklılığının ortalama standart sapma değeri 3,87 olduğu ve görüntüler arasında gözle ayırt edilebilir bir fark olmadığı görülmüştür. Test sonuçlarında Jaccard Index'e göre IsoData ve Otsu algoritmalarının ortalamada en iyi benzerlik oranına ve en iyi ikili sınıflandırma başarısına sahip olduğu görülmüştür. Elbette edilen bulgular, diğer algoritmaların daha düşük benzerlik oranına sahip olduğu için kötü olduğu anlamına gelmemelidir. Her biri spesifik çözümler için tercih edilebilecek potansiyele sahip yöntemlerdir.

\section{KAYNAKÇA}

[1] Kotte, S., Rajesh Kumar, P., \& Injeti, S. K., "An efficient approach for optimal multilevel thresholding selection for gray scale images based on improved differential search algorithm", Ain Shams Engineering Journal, vol. 9, no. 4, pp. 1043-1067, 2018.

[2] Wang, Z., Wang, E., \& Zhu, Y., "Image segmentation evaluation: a survey of methods", Artificial Intelligence Review., 2020.

[3] Ramola, A., Shakya, A.K., \& Pham, D.V., "Study of statistical methods for texture analysis and their modern evolutions", Engineering Reports, vol. 2, no. 4, pp. 1-24, 2020.

[4] Aqeel, E. F., "The Use of Threshold Technique in image segmentation", Journal of the College of Basic Education, vol. 21, no. 89, pp. 1-12, 2015.

[5] Zaitoun, N.M., \& Aqel, M.J., "Survey on Image Segmentation Techniques", Procedia Computer Science, vol. 65, pp. 797-806, 2015.

[6] Singh, T.R., Roy, S., Singh, O.I., Sinam, T., \& Singh, Kh.M., "A New Local Adaptive Thresholding Technique in Binarization", International Journal of
Computer Science Issues, vol. 8, no. 2, pp.271-277, 2011.

[7] Ceylan, R., \& Koyuncu, H., "ScPSO-Based Multithresholding Modalities for Suspicious Region Detection on Mammograms", Soft Computing Based Medical Image Analysis, Academic Press, pp. 109135, 2018.

[8] Rosin, P., "Thresholding for change detection". Sixth International Conference on Computer Vision (IEEE Cat. No.98CH36271), pp. 274-279, 1998.

[9] Ekielski, A., "An algorithm for determination of threshold value in extruded products by the method of maximum increments: modification of Otsu method", Annals of Warsaw University of Life Sciences-SGGW, Agriculture no. 62, pp. 71-79, (2013).

[10] Sezgin, M. \& Sankur, B., "Survey over Image Thresholding Techniques and Quantitative Performance Evaluation", Journal of Electronic Imaging, vol. 13, no. 1, pp. 146-165, 2004. 
[11] Otsu, N., "A threshold selection method from graylevel histograms", IEEE Trans. Sys., Man., Cyber, vol. 9, pp. 62-66, 1979.

[12] Doyle, W., "Operation useful for similarity-invariant pattern recognition", Journal of the Association for Computing Machinery vol. 9, pp. 259-267, 1962.

[13] Kittler, J., Illingworth, J. and Foglein, J., "Threshold selection based in a simple image statistic", Computer Vision, Graphics and Image Processing, vol. 30, pp. 125-147, 1985.

[14] Ridler, T. W. \& Calvard, S., "Picture thresholding using an iterative selection method", IEEE Transactions on Systems, Man and Cybernetics, vol. 8, pp. 630-632, 1978.

[15] Glasbey, C. A., "An analysis of histogram-based thresholding algorithms", CVGIP: Graphical Models and Image Processing, vol. 55, pp. 532-537, 1993.

[16] Kapur, J. N., Sahoo, P. K. \& Wong, A. C. K., “A New Method for Gray-Level Picture Thresholding Using the Entropy of the Histogram", Graphical Models and Image Processing, vol. 29, no. 3, pp. 273-285, 1985.

[17] Sahoo, P., Wilkins, C. \& Yeager, J., "Threshold selection using Renyi's entropy", Pattern Recognition, vol. 30, no. 1, pp. 71-84, 1997.

[18] Li, C. H. \& Lee, C. K., "Minimum Cross Entropy Thresholding", Pattern Recognition, vol. 26, no. 4, pp. 617-625, 1993.
[19] Li, C. H. \& Tam, P. K. S., "An Iterative Algorithm for Minimum Cross Entropy Thresholding", Pattern Recognition Letters, vol. 18, no.8, pp. 771-776, 1998.

[20] Shanbhag, Abhijit G., "Utilization of information measure as a means of image thresholding", Graph. Models Image Process. (Academic Press, Inc.), vol. 56, no. 5, pp. 414-419, 1994.

[21] Yen J. C., Chang F. J., Chang S., "A New Criterion for Automatic Multilevel Thresholding", IEEE Trans. on Image Processing, vol. 4, no. 3, pp. 370-378, 1995.

[22] Zack, G. W., Rogers, W. E., Latt, S. A., “Automatic measurement of sister chromatid exchange frequency", J. Histochem. Cytochem, vol. 25, no. 7, pp. 741-53, 1977.

[23] Prewitt, J. M. S. \& Mendelsohn, M. L., "The analysis of cell images", Annals of the New York Academy of Sciences, vol. 128, pp. 1035-1053, 1966.

[24] Tsai, W., "Moment-preserving thresholding: a new approach", Computer Vision, Graphics, and Image Processing, vol. 29, pp. 377-393, 1985.

[25] Huang, L-K. \& Wang, M-J. J., "Image thresholding by minimizing the measure of fuzziness", Pattern Recognition, vol. 28, no. 1, pp. 41-51, 1995.

[26] Stelios, K., Michail, K., \& Vassilios, C., "An Empirical Method for Threshold Selection", International Journal of Signal Processing, Image Processing and Pattern Recognition, vol. 5, no. 2, pp. 101-114, 2012. 